\title{
Induction of male production in clones of Daphnia pulex by the juvenoid hormone methyl farnesoate under short photoperiod
}

\author{
Winfried Lampert ${ }^{\text {a,b,c,*}, ~ K a t h r i n ~ P . ~ L a m p e r t ~}{ }^{c}$, Petter Larsson ${ }^{\text {a }}$ \\ a University of Bergen, Department of Biology, Postbox 7803, N-5020 Bergen, Norway \\ b Max Planck Institute for Evolutionary Biology, Postfach 165, 24302 Plön, Germany \\ c University of Bochum, Evolutionary Ecology and Biodiversity of Animals, Universitätsstr. 150, 44780 Bochum, Germany
}

\section{A R T I C L E I N F O}

\section{Article history:}

Received 9 March 2012

Received in revised form 22 May 2012

Accepted 22 May 2012

Available online 26 May 2012

\section{Keywords:}

Daphnia

Clonal diversity

Male production

Methyl farnesoate

Photoperiod

\begin{abstract}
A B S T R A C T
The juvenile hormone, methyl farnesoate (MF), and its analog insecticides have been used successfully to induce the production of males in cladocerans under long-day conditions in the laboratory. However, without hormone addition Daphnia do not usually produce male offspring under long photoperiods, while short photoperiods are a stimulus for the induction of males. We used 21 clones of Daphnia pulex differing in their propensity to produce males under short-day conditions to test whether the treatment with MF would result in an additive effect of shifting the sex ratio towards males. Contrary to our expectations, clones with a high tendency of male production showed a reduced sex ratio in response to MF treatment under short-day conditions, but clones that produced normally few males or did not produce males were stimulated by $700 \mathrm{nM}$ MF to produce up to $40 \%$ males. We suggest that the endocrine disruptive effect of MF or juvenile hormone analogs in the field may depend on the clonal composition of the cladoceran population and on the natural photoperiod. This may affect the seasonal occurrence of sexual reproduction and eventually cause a mismatch between the presence of males and ephippial females.
\end{abstract}

(c) 2012 Elsevier Inc. All rights reserved.

\section{Introduction}

The production of males in the cyclic parthenogenetic Daphnia is induced by environmental stimuli such as short photoperiod and crowding (see review in Hobaek and Larsson, 1990). Light period is a particularly important regulator of the sex ratio, i.e. the proportion of males produced is suppressed under long-day conditions (Innes and Dunbrack, 1993; Deng, 1996). Deng (1996) reported a critical light period of $14 \mathrm{~h}$ of light per day. Environmental cues may trigger the expression of transcripts associated with endocrine regulation (Eads et al., 2008) affecting the hormonal pathways controlling physiological processes. The terpenoid juvenile hormone, methyl farnesoate (MF), is particularly important in crustacean sex determination (LeBlanc, 2007). Daphnia has been shown to shift the sex ratio in favor of males when exposed to MF in the water (Olmstead and LeBlanc, 2002). This has become an interesting topic for ecotoxicology as other juvenile hormones and their analogs, hormone-mimicking insecticides, have been found to have similar effects (Oda et al., 2005, 2007) and are suspected to be endocrine disrupters (Olmstead and LeBlanc, 2003).

* Corresponding author at: Max Planck Institute for Evolutionary Biology, Postfach 165, 24302 Plön, Germany. Tel.: + 494522763 270; fax: + 494522763310. E-mail addresses: lampert@evolbio.mpg.de (W. Lampert)

kathrin.lampert@ruhr-uni-bochum.de (K.P. Lampert), petter.larsson@bio.uib.no (P. Larsson)
In some cladoceran species and clones with a very low propensity to produce males, MF has also been used as a tool to increase the sex ratio in Daphnia. For example, applying MF Kim et al. (2006) were able to induce males that so far had been unknown to science in some lacustrine cladocerans. Ikuno et al. (2008) produced large numbers of males of Daphnia magna to test differences in chemical stress responses between male and female neonates. It is to be expected that MF will be used more frequently in Daphnia genetics to produce males for crossing experiments.

Typical MF experiments have been carried out with single clones of cladocerans. However, Oda et al. (2006) found differences in the sex ratios of three strains of $D$. magna in response to two juvenile hormone analogs. This was to be expected as the clonal variability in traits of sexual reproduction can be considerable (Larsson, 1991). In addition, the experiments were performed under long-day conditions (continuous light; L:D cycles of $16: 8$ or $14: 10 \mathrm{~h}$ ) that are normally used to suppress the production of males. As field populations consist of a large variety of clones and light conditions change seasonally, there may be difficulties to apply the typical laboratory results to field conditions. When we ran experiments to test the expression of traits of sexual reproduction among many clones (Lampert et al., 2012), we also used MF in addition to short-day conditions, a known stimulus of male production. Our hypotheses were that clonal differences of the sex ratio existed in response to MF, and that MF had an additive effect to a short photoperiod as stimulus. We hoped that by exposure to MF we would be able to estimate the full potential 
of male production of the individual clones independently of a varying importance of environmental stimuli.

\section{Material and methods}

Experiments were performed with 21 clones of Daphnia pulex from a small, mesotrophic lake (Myravann) near the town of Bergen, Norway. The clones had been isolated between November 2006 and March 2007. Eleven clones were offspring of females collected in the open water, while 10 clones originated from ephippial, dormant embryos collected from the sediments and hatched in the laboratory (Lampert et al., 2010). These clones had been kept in the laboratory for more than two years at $20^{\circ} \mathrm{C}$ under continuous light to suppress sexual reproduction. Stock cultures as well as experimental animals were maintained in membrane-filtered $(0.45 \mu \mathrm{m})$ lake water from Myravann, and they were fed green algae (Scenedesmus obliquus) grown in chemostats (Lampert et al., 2010).

Females were treated with MF shortly before they reached maturity, i.e. when they started to accumulate yolk in the ovary but did not have eggs in the brood pouch yet (Olmstead and LeBlanc, 2002). Some large egg-carrying females of each clone were isolated in $300 \mathrm{~mL}$ jars, and neonates were collected as soon as they had been released. For each clone, $6 \times 5$ neonates were combined in $50 \mathrm{~mL}$ glass jars containing food suspension of $1 \mathrm{mg} \mathrm{C} \mathrm{L}^{-1}$. They were kept at $20^{\circ} \mathrm{C}$ under short-day ( $8 \mathrm{~h}$ light) conditions and transferred to fresh containers every day. After four days, when the females had reached approximately $1.5 \mathrm{~mm}$ length, the numbers were reduced to 3 per jar. Under these conditions (daily removal of the medium), competition for food was not important as control females reached an average of 7 eggs in their first clutch, which comes close to the numbers obtained under optimal conditions in a flow-through system (W. Lampert, unpubl. results). Although we cannot rule out an additional effect of crowding on male production with 3 individuals in $50 \mathrm{~mL}\left(60 \mathrm{~L}^{-1}\right)$ it is unlikely as this density is below the density in earlier experiments on male induction (Larsson, 1991; Kleiven et al., 1992). Then three jars of each clone were exposed to a nominal concentration of $700 \mathrm{nM}$ (E,E) MF (Echelon, S-0153) with $0.1 \%$ ethanol as solvent (Oda et al., 2006). This has been shown to be the saturation concentration for male production in Daphnia pulicaria, closely related to D. pulex (Kim et al. 2006). After three initial days, the MF concentration was reduced to $233 \mathrm{nM}$. The remaining three jars served as controls and they received only the solvent. Females were transferred to new medium daily until the first neonates in a jar had been released. This happened on average three days later in the treatments than in the controls. For the successive four days females were transferred every second day. This resulted in three samples of neonates from each jar, originating from the first and second clutch. After adult females had been transferred, neonates were kept in the jars for five days to let them grow and make sex determination more reliable. They were then preserved and sexed later under a dissecting microscope. To compensate for the loss of individual adults during the experiment we pooled offspring from the three jars and the three sampling dates and calculated the mean proportion of males in the neonate population, and the mean number of offspring (females and males) per female.

\section{Results}

Under short-day conditions, individual clones showed great variability in the production of males. The proportion of male offspring varied between 0 and 43\% in the controls (Fig.1) and between 0 and $51 \%$ in the MF treatments. However, all 21 clones produced males in at least one situation, either in the controls (17) or in the MF treatments (15). Hence none of the clones can be considered an obligate parthenogens. Mean $( \pm S D)$ percentages of males in the controls and treatments were $17.29 \pm 15.03$ and $9.83 \pm 12.93$, respectively.

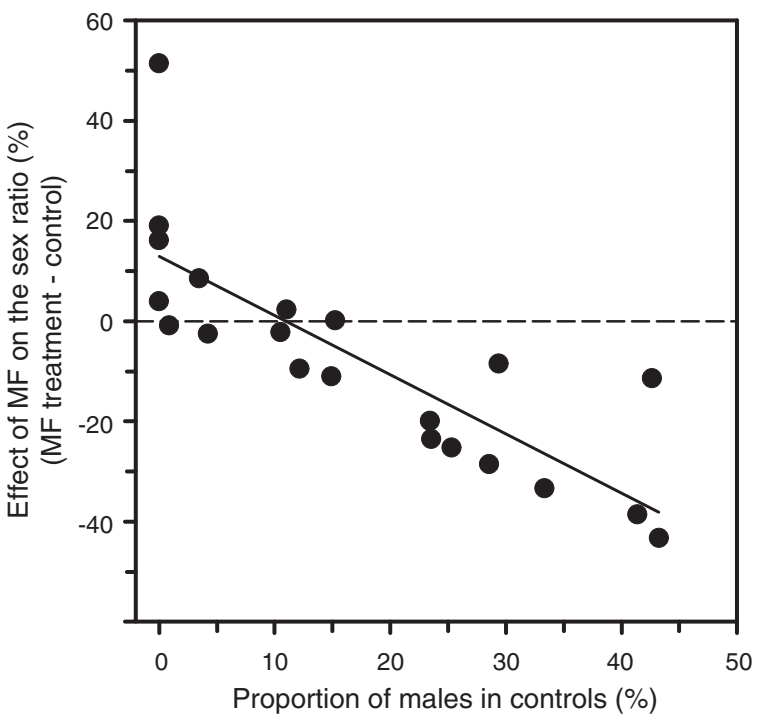

Fig. 1. Effect of methyl farnesoate on individual clones of Daphnia pulex at a short photoperiod. Differences between sex ratios (\% of male neonates) in MF treatments and controls ([\% males in treatment $]-[\%$ males in control $]$ ). Each dot represents a single clone.

Due to the large SD, the difference between groups was not significant, neither in a $t$-test. $(t=1.724, p=0.046)$ nor in a non-parametric test (Mann-Whitney U).

Although individual clones varied in their response to MF, a pattern emerged when the MF effect was quantified for each clone as the difference between male proportions in MF treatments and controls (Fig. 1). MF enhanced the male production only in clones that had very low male proportions in the controls, in particular in all clones that produced no males at all in the absence of MF. On the contrary, male production was suppressed by MF in clones that showed a strong propensity for male production in the controls. Consequently, the average effect of MF was negative. A linear regression of the MF effect in response to the tendency of clones to produce males without additional MF (Fig. 1) was significant $(\mathrm{y}=12.96-1.18 \mathrm{x}$, $\left.r^{2}=0.664, p<0.001\right)$.

In addition, MF had a negative effect on the total offspring (females + males) production and on the growth rate of females (Table 1$)$. The mean number $( \pm S D)$ of neonates per female in the controls and in the treatments was $9.05 \pm 2.95$ and $2.96 \pm 1.85$, respectively, and this difference was highly significant $(t=8.025$, $p>0.001)$. At the time of release of their first clutch, females in MF treatments were significantly $(t=17.05, p<0.001)$ older by 5.5 days on average. However, there was no correlation between the effects of MF on the proportion of males and the number of offspring. Neither the proportion of males in the controls nor the effect of MF on the proportion of males was correlated with neonate production.

\section{Discussion}

This study was associated to a larger experiment that measured the tendency to produce males in different clones of $D$. pulex as an adaptive trait related to overwintering strategies (Lampert et al.,

Table 1

Effects of methyl farnesoate on reproductive characteristics (mean $\pm S D$ ) of 21 clones of Daphnia pulex.

\begin{tabular}{lcc}
\hline & Control & Treatment \\
\hline Neonates per female & $9.05 \pm 2.95$ & $2.96 \pm 1.85$ \\
Age at release of the first clutch (d) & $9.55 \pm 0.95$ & $15.07 \pm 1.14$ \\
Proportion of male offspring (\%) & $17.29 \pm 15.03$ & $9.83 \pm 12.93$ \\
\hline
\end{tabular}


2012). We suspected that treatment with the juvenoid hormone MF that has been shown to enhance male production (Olmstead and LeBlanc, 2002, 2003; Matsumoto et al., 2008) might reveal an estimate of full potential of clone-specific male production. Hence we applied the minimum MF concentrations reported to result in the maximum effect on the sex ratio (Olmstead and Leblanc, 2003; Kim et al., 2006). However, as we attempted an ecological rather than a physiological or ecotoxicological approach, our study differs from earlier work in several respects: (1) we tested the clonal variability of the sex ratio in response to MF; (2) we tested the effect of MF under short-day conditions, a natural cue to induce male production (Innes and Dunbrack, 1993; Deng, 1996); (3) we report the effect of MF on growth and offspring production.

As expected (Larsson, 1991; Innes and Dunbrack, 1993; Deng, 1996), the production of males in the controls (without MF treatment) differed considerably among clones. The clonal variability of male production in the MF treatments was in the same range as in the controls. Although systematic studies of genetic differences in the susceptibility to MF are still missing, it has been demonstrated that three (Oda et al., 2006) and seven (Oda et al., 2007) clones of $D$. magna differed with respect to the production of male neonates in response to fenoxycarb, a juvenile hormone analog.

Contrary to our expectations, the average sex ratio was not enhanced in the MF treatments under short-day conditions. Consequently, there was no additive effect of MF compared to the short photoperiod alone. Rather the effect seems to be cumulatively toxic as a clear pattern emerged. Only clones with a very low tendency of male production in the controls showed enhanced male production with MF. On the contrary, MF suppressed the proportion of males in clones that had already a high propensity of male production in the controls (Fig. 1). To our knowledge, this has not been reported before.

The mechanism of this effect is not clear but we may speculate that it is related to the multiple functions of MF in the endocrine gene regulation (LeBlanc, 2007). If MF is involved in the regulation of sex determination we can assume that the sex ratio is correlated with a certain internal level of MF. Long-day conditions suppress the production of males in Daphnia, i.e. a long photoperiod may be related to a low internal MF level. Short-day conditions induce male production but there are clonal differences in intensity, i.e. internal MF level. Clones that show low sex ratios despite a short photoperiod may need an additional stimulus (Kleiven et al., 1992). External MF addition increases the internal MF level, and thus promotes the production of males in individuals that naturally had low internal MF levels. This assumption is consistent with the regularly enhancing effect of MF on sex ratios in long-day experiments as well as with the increased sex ratio only for clones that showed zero or low male production under short-day conditions. However, if females have already a high internal MF level exogenous application of MF may raise the internal level too much, which disturbs the regulation of the hormone circuit (LeBlanc, 2007), causes a classical endocrine negative feedback on internal MF levels, affects growth and molting, and may eventually reduce the sex ratio.

The multiple functions of MF in the hormone circuit (LeBlanc, 2007) may also be responsible for the lowered growth rate and neonate production in our MF treatments. Although most studies with MF report only the sex ratio, but not the total number of offspring, toxicological reproduction tests with juvenile hormone analogs under long-day conditions found reduced neonate production. Oda et al. (2007) warned that the effect of fenoxycarb on the sex ratio of $D$. magna in chronic (21 days) tests can be obscured due to very low reproductive rates. We found significantly reduced mean neonate production of $D$. pulex in the presence of MF even under short-day conditions, although we considered only the first clutch and parts of the second.

MF has been a helpful tool to detect and describe males of cladoceran species that exhibit a low tendency to sexual reproduction (Kim et al., 2006). This is consistent with our finding of a stronger response to MF treatment in clones with a low production of males. Although Rider et al. (2005) seem to report different results their study does not contradict our conclusions. Rider et al. (2005) exposed two groups of $D$. pulex/pulicaria to MF under a long photoperiod (16:8 h L:D). The first group has been reported to produce males. However, five of the six clones did not produce any males in the long-day controls. But when treated with MF, five clones showed significantly enhanced sex ratios. This supports earlier reports that long-day conditions suppress male production.

The second group consisted of six clones that had been observed to never produce males under "natural" conditions. Evidently these were obligate parthenogenetic clones (Innes and Dunbrack, 1993). Consequently, none of them produced males, neither in the MF treatments nor in the controls. Rider et al. (2005) concluded that these clones lacked the MF receptor. We did not find obligate parthenogens among our 21 clones. Although some of them produced no males either in the controls or in the MF treatments, not a single clone showed a zero sex ratio in both treatments under short photoperiod. Hence, all our clones had the capacity to produce males but differed in sensitivity.

In toxicology, MF has been used to produce sufficient numbers of males in comparative studies on the sensitivity to chemical compounds of females and males of $D$. magna (Ikuno et al., 2008). However, our study calls for caution in the interpretation of experiments on the endocrine disruptive activity of juvenile hormone analogs in the environment. The large clonal differences in the response to MF should be kept in mind if laboratory experiments are being extrapolated to the field, and more studies on clonal variability may be needed for other compounds. In addition, hormone analogs in the field may act differently during different seasons. In periods when environmental cues favor the production of males anyway, additional hormone analogs may suppress the production of males, while they may increase the sex ratio at times when no natural male production occurs. This may disturb the synchronization between male production and sexual egg production that normally leads to pulses of ephippia in a lake (Caceres and Tessier, 2004).

\section{Conclusions}

We found that day length modifies the effect of exposure to the juvenoid hormone methyl farnesoate on male production by Daphnia. MF has been reported to be involved in regulating the switch of Daphnia from the parthenogenetic to the sexual mode of reproduction. It is long known that under natural conditions the male production of Daphnia is strongly depressed by long photoperiods. As the male production of Daphnia under long photoperiods can be enhanced by exposure to MF, it seems that long photoperiods suppress the internal level of MF. Under short photoperiods, clones of Daphnia exhibit large differences in sex ratios, i.e. they probably have different internal levels of MF. We found that the exposure of Daphnia clones to MF did not have a general additive effect on the sex ratio. Rather the exposure to MF had a stimulating effect on the sex ratio of clones with a naturally low propensity of male production (proposed low internal MF level), while it affected the sex ratio of clones with a naturally high propensity to produce males (proposed high internal level of MF) negatively, probably a toxic effect. Hence the photoperiod should be considered when assessing the effect of MF in the environment.

\section{Acknowledgments}

This paper reports results of a cooperative project between the University of Bergen, Norway, the University of Bochum, Germany, and the Max Planck Institute of Evolutionary Biology at Plön, Germany. The project was made possible by the temporary employment of W. L. as Professor II by the University of Bergen. We are grateful to Knut-Helge 
Jensen for support in the laboratory and maintaining the Daphnia clones, and to Werner Kloas for endocrinological advice and encouragement to publish these results. We appreciate the constructive comments of two anonymous reviewers that helped to improve a first version of this manuscript. Financial support came from the Melzer Foundation and the Max Planck Society for the Advancement of Science. The genetic characterization of the Daphnia clones was funded by a grant of the Deutsche Forschungsgemeinschaft (La 1382/3-1) to K. P. L.

\section{References}

Caceres, C.E., Tessier, A.J., 2004. Incidence of diapause varies among populations of Daphnia pulicaria. Oecologia 141, 425-431.

Deng, H.-W., 1996. Environmental and genetic control of sexual reproduction in Daphnia. Heredity 76, 449-478.

Eads, B.D., Andrews, J., Colbourne, J.K., 2008. Ecological genomics in Daphnia: stress responses and environmental sex determination. Heredity 100, 184-190.

Hobaek, A., Larsson, P., 1990. Sex determination in Daphnia magna. Ecology 71 2255-2268.

Ikuno, E., Matsumoto, T., Okubo, T., Itoi, S., Sugita, H., 2008. Difference in the sensitivity to chemical compounds between female and male neonates of Daphnia magna. Environ. Toxicol. 23, 570-575.

Innes, D.J., Dunbrack, R.L., 1993. Sex allocation variation in Daphnia pulex. J. Evol. Biol. 6, 559-575.

Kim, K., Kotov, A.A., Taylor, D.J., 2006. Hormonal induction of undescribed males resolves cryptic species of cladocerans. Proc. R Soc. Lond. B 273, 141-147.

Kleiven, O.T., Larsson, P., Hobaek, A., 1992. Sexual reproduction in Daphnia magna requires three stimuli. Oikos $65,197-206$
Lampert, W., Lampert, K.P., Larsson, P., 2010. Coexisting overwintering strategies in Daphnia pulex: a test of genetic differences and growth responses. Limnol. Oceanogr. 55, 1893-1900.

Lampert, W., Lampert, K.P., Larsson, P., 2012. Coexisting overwintering strategies in Daphnia pulex: clonal differences in sexual reproduction. Fundam. Appl. Limnol. 179, 281-291.

Larsson, P., 1991. Intraspecific variability in response to stimuli for male and ephippia formation in Daphnia pulex. Hydrobiologia 225, 281-290.

LeBlanc, G.A., 2007. Crustacean endocrine toxicology: a review. Ecotoxicology 16, 61-81.

Matsumoto, T., Ikuno, E., Itoi, S., Sugita, H., 2008. Chemical sensitivity of the male daphnid, Daphnia magna, induced by exposure to juvenile hormone and its analogs. Chemosphere 72, 451-456.

Oda, S., Tatarazako, N., Watanabe, H., Morita, M., Iguchi, T., 2005. Production of male neonates in Daphnia magna (cladocera, crustacea) exposed to juvenile hormones and their analogs. Chemosphere 61, 1168-1174.

Oda, S., Tatarazako, N., Watanabe, H., Morita, M., Iguchi, T., 2006. Genetic differences in the production of male neonates in Daphnia magna exposed to juvenile hormone analogs. Chemosphere 63, 1477-1484.

Oda, S., Tatarazako, N., Dorgerloh, M., Johnson, R.D., Kusk, K.O., Leverett, D., Marchini, S., Nakari, T., Williams, T., Iguchi, T., 2007. Strain difference in sensitivity to 3,4dichloroaniline and insect growth regulator, fenoxycarb, in Daphnia magna. Ecotoxicol. Environ. Saf. 67, 399-405.

Olmstead, A.W., Leblanc, G.A., 2002. Juvenoid hormone methyl farnesoate is a sex determinant in the crustacean Daphnia magna. J. Exp. Zool. 293, 736-739.

Olmstead, A.W., LeBlanc, G.A., 2003. Insecticidal juvenile hormone analogs stimulate the production of male offspring in the crustacean Daphnia magna. Environ. Health Perspect. 111, 919-924.

Rider, C.V., Gorr T.A., Olmstead, A.W., Wasilak, B.A., LeBlanc, G.A. 2005. Stress signaling: coregulation of hemoglobin and male sex determination through a terpenoid signaling pathway in a crustacean. J. Exp. Biol. 208, 15-23. 